\title{
THE ALVEOLAR SEPTAL THICKNESS AND TYPE II PNEUMOCYTES NUMBER IN IRRADIATED LUNGS, TIME EXPRESSION AND THE EFFECT OF PENTOXIFYLLINE
}

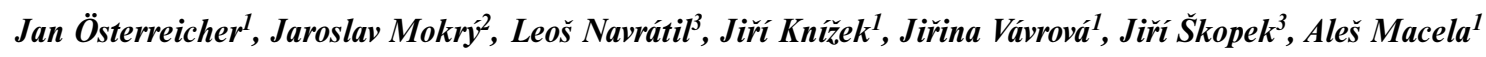

Purkyně Military Medical Academy in Hradec Králové: Department of Radiobiology and Immunology ${ }^{1}$; Charles University in Prague, Faculty of Medicine in Hradec Králové: Department of Histology and Embryology²; Charles University in Prague, $1^{\text {st }}$ Medical Faculty: Department of Biophysics ${ }^{3}$

\begin{abstract}
Summary: Purpose: We studied the relationship between type II pneumocytes number and alveolar septal thickness during different time after sublethal whole-thorax irradiation of rats and we investigated the influence of pentoxifylline (TNF- $\alpha$ inhibitor). Materials and methods: Wistar rats were exposed to $15 \mathrm{~Gy}$ thoracic irradiation and pentoxifylline $(35 \mathrm{mg} / \mathrm{kg})$ twice a week. Lungs were examined histologically and imunohistochemically at intervals ranging from 1-12 weeks and alveolar septal thickness, number of type II pneumocytes (identified by immunoreactivity for cytokeratin 18), and neutrophile granulocytes were counted. Results: Significant increase of alveolar septal thickness and type II pneumocytes depletion 3 weeks after irradiation were found. Correlation of these markers was $r=-0,759$. Pentoxifylline significantly inhibits increased alveolar septal thickness without the influence on type II pneumocytes number. Neutrophil penetration started 5 weeks after irradiation in non-treated animals, 8 weeks after irradiation in PTX-treated rats. Conclusions: We suggest that pneumocytes depletion is linked to increased vascular permeability, and pentoxifylline therapy does not influence on pneumocytes kinetics after irradiation.
\end{abstract}

Key words: $\gamma$-Irradiation; Lung; Pentoxifylline; Pneumocytes type II; Interstitial edema

Abbreviations: ARDS - Adult Respiratory Distress Syndrome; CK 18 - cytokeratin 18; DAD - diffuse alveolar damage; DXM - Dexamethasone; IFN- $\gamma$ - Interferone gamma; IL - Interleukin; KGF - Keratinocyte Growth Factor; mRNA - messenger ribonucleic acid; PBS - phosphate-buffered saline; PTX - Pentoxifylline; RP - Radiation pneumonitis; S.E.M - standard error of mean; TNF - Tumor necrosis factor.

\section{Introduction}

Radiation pneumonitis (RP) is defined as an exudative inflammation which occurs as a result of ionizing radiation (3) and it is considered as an alveolitis resulting from the damage of the pneumocytes and endothelial cells (10). This condition is termed Adult Respiratory Distress Syndrome (ARDS) or Diffuse Alveolar Damage (DAD; 11). RP usually starts from 2 to 6 months after irradiation of the lungs with a single dose of $7 \mathrm{~Gy}$ and higher (1). It involves the changes in the irradiated areas only (22).

The main histologic features of RP are interstitial edema and the presence of inflammatory cells in irradiated tissue. Interstitial lung edema intensity can be determined by alveolar septal thickness measurement (17). In early time interval, 3 weeks after a whole-thorax irradiation, increased vascular permeability with slightly thickened alveolar septa in irradiated lungs was described $(3,10,19)$. Mechanisms of this phenomenon are still unclear and there exist sug- gestion that increased pulmonary vascular permeability may be caused by endothelial cell damage (10) or alveolar cells alteration (12).

Pentoxifylline (PTX) ameliorates radiation-induced histological changes in irradiated lungs during RP manifestation (18). In clinical practice, PTX is used mainly as a vasodilatator. Funk et al. (6) observed in vitro influence of cytokine production after the addition of PTX alone and PTX in combination with DXM in their experiments with human peripheral mononuclear cells, which were stimulated by phytohemaglutinin. These results suggest that PTX inhibits the production of TNF- $\alpha$, IL-2, and IFN- $\gamma$, known as proinflammatory cytokines. The proinflammatory cytokines and adhesion molecules inhibition due to PTX therapy confirmed Silva et al. (21), Noel et al. (16), and Koo et al. (15).

In this study we sought a determined the relationship between markers as type II pneumocytes number detected by cytokeratin 18 (CK 18) (25) and alveolar septal thick- 
ness as morphological indicator of interstitial edema during early time intervals, due to increased vascular permeability. We also investigated the effect of pentoxifylline administered from day 1 to day 84 (12 weeks) after irradiation on alveolar septal thickness and type II pneumocytes number.

\section{Material and Methods}

Male Wistar rats (Konarovice, Czech Republic) aged 8-12 weeks weighing 150-200 g were exposed to local thoracic irradiation using a ${ }^{60} \mathrm{Co}$ unit (Chisotron Chirana) at a dose rate of $1.0 \mathrm{~Gy} / \mathrm{min}$, target distance $1 \mathrm{~m}$. The animals were slightly anaesthetised before irradiation using a mixture of one volume of Rometar (Spofa, Prague), 3 volumes of Narkamon (Léčiva, Prague) and 12 volumes of physiological saline. This solution was injected intramuscularly at a dose of $10 \mathrm{ml} / \mathrm{kg}$. Local thoracic irradiation was performed in a jig in which a $10 \mathrm{~cm}$ thick layer of lead reduced the dose to other parts of the body to around $2-3 \%$ of the lung dose (26).

Three groups of rats were used: Group $\mathrm{S}$ was given physiological saline only. Group PTX 35 was given pentoxiphylline (Pentilin, Krka, $100 \mathrm{mg} / 5 \mathrm{ml}$ ampules) diluted with physiological saline and administered at a dose of $35 \mathrm{mg} / \mathrm{kg}$. Group C involved sham-irradiated controls.

PTX and saline were injected subcutaneously twice a week; each of these three groups included 7 subgroups with a total of 42 rats irradiated with 15 Gy and treated at $1,2,3,4,5,8$, and 12 weeks after irradiation. This radiation dose resulted in significant pneumonitis and fibrosis but no mortality up to 30 weeks (26), whereas 16 Gy resulted in over $50 \%$ mortality 6 weeks after irradiation. The first drug administration was given $2 \mathrm{~h}$ after irradiation and the last at two days before the animals were sacrificed.

\section{Histological Examination}

Rats were killed by cervical dislocation and lung histology examined at 1, 2, 3, 4, 5, 8 and 12 weeks after irradiation; controls were killed at 12 weeks. Marker levels measured in 6 control rats were used as reference values. During dissection, the lungs were carefully fixed per tracheam with $10 \%$ neutral buffered formalin, embedded into paraffin and $1 \mu \mathrm{m}$ thick tissue sections were cut, stained with haematoxylin-eosin for alveolar septal thickness measurement, for chloroacetate esterase to detect neutrophil granulocytes and Gram's staining was used for bacterial infection.

Immunohistochemical examination of CK 18 was performed with a standardized immunoperoxidase technique. After blocking of endogenous peroxidase activity for $20 \mathrm{~min}$, tissue sections were incubated with monoclonal antibody recognising cytokeratin 18 (clone C-04, Exbio, Prague, Czech Republic; 25) diluted 1:5 in phosphate-buffered saline (PBS, $\mathrm{pH}$ 7.2) for $1 \mathrm{~h}$, and then washed three times in PBS. Slides were then incubated with a biotinyla- ted anti-mouse antibody (Biogenex, Prague, Czech Republic) for 45 minutes. Subsequently, slides were incubated with streptavidin horseradish peroxidase (Biogenex, Prague, Czech Republic) under the same conditions as with the secondary antibody. Excess antibody was washed off with PBS. Finally, 0.05\% 3,3-diaminobenzidine tetrahydrochloride chromogen solution (Sigma, Prague, Czech Republic) in PBS containing $0.02 \%$ hydrogen peroxide was added for $10 \mathrm{~min}$ to visualise the antigen-antibody complex in situ.

\section{Measurement of alveolar septal thickness, type II} pneumocytes number, and neutrophils number

Slides stained with anti-cytokeratin 18 for type II pneumocytes and haematoxylin-eosin were evaluated using an IMT-2 light microscope (Olympus Company, Prague) and computer image analysis (Image Pro, Media Cybernetics, MD, USA). Ten randomly selected viewing fields from each sample stained with anti-cytokeratin 18 with a size of $10,744.32 \mu \mathrm{m}^{2}$ were evaluated in each slide at original magnification x 600. Only whole-imaged alveoli were counted in each field; areas containing partially-imaged alveoli, bronchi and large vessels were excluded from analysis. Cells immunoreactive for cytokeratine 18 ( CK 18+) in counted alveoli were detected in the range red 104-170, green 108-152, and blue 108-140, where 0 is black colour and 256 is white colour.

For alveolar septal thickness measurement 6 randomly selected viewing fields of the same size and magnification were evaluated in each slide. In each viewing field 10 randomly selected alveolar septa were measured in the narrowest point of their length (17).

In each slide stained for chloroacetate esterase, neutrophils were identified using the Amplival light microscope (Carl Zeiss, Jena). Fifteen viewing fields were examined at original magnification x $640\left(282,743.3 \mu \mathrm{m}^{2}\right.$ per field; 18).

\section{Data Processing}

The Mann-Whitney test was used for statistical analysis giving mean $\pm 2 \times$ S.E.M. The correlation coefficient between alveolar septal thickness and CK 18+ cells values was determined.

\section{Results}

In Group S, 3 animals died, 2 at 4 weeks and 1 at 12 weeks after irradiation. One rat from the PTX-administered group died at 12 weeks after irradiation. No infectious agents were found.

\section{The alveolar septal thickness measurement}

Significantly lower values of Group C compared with Group $\mathrm{S}$ were measured within each time interval except week 2 and week 5 after irradiation. In Group PTX 35 compared with Group C, significantly higher values were seen 
Tab. 1: Average number of CK 18+ cells per alveolus in percents $\pm 2 \times$ SEM.

\begin{tabular}{|l|c|c|c|c|c|c|c|}
\hline & 1 week & 2 weeks & 3 weeks & 4 weeks & 5 weeks & 8 weeks & 12 weeks \\
\hline Group S & $6,75 \pm 4,79$ & $5,62 \pm 2,90$ & $2,71 \pm 2,69^{3}$ & $5,91 \pm 1,56$ & $8,02 \pm 2,21$ & $5,98 \pm 3,55$ & $4,59 \pm 2,04^{2}$ \\
\hline Group PTX 35 & $11,37 \pm 2,98$ & $10,10 \pm 4,78$ & $5,47 \pm 1,60$ & $6,96 \pm 1,76$ & $7,40 \pm 1,96$ & $7,70 \pm 2,39$ & $5,91 \pm 2,10$ \\
\hline Group C & & & & & & & $9,31 \pm 3,59$ \\
\hline
\end{tabular}

Probability of values difference to Group C: $\mathrm{p}<0,05-{ }^{1} ; \mathrm{p}<0,01-{ }^{2} ; \mathrm{p}<0,001-{ }^{3}$.

Probability of values difference between Group S and Group PTX 35 in the same time interval: $p<0,05-{ }^{a} ; p<0,01-{ }^{b}$; $\mathrm{p}<0,001-\mathrm{c}$

Tab. 2: Average alveolar septal thickness $\pm 2 \times$ SEM.

\begin{tabular}{|l|c|c|c|c|c|c|c|}
\hline & 1 week & 2 weeks & 3 weeks & 4 weeks & 5 weeks & 8 weeks & 12 weeks \\
\hline Group S & $4,84 \pm 0,20^{3}$ & $4,19 \pm 0,19$ & $6,39 \pm 0,29^{3}$ & $5,38 \pm 0,29^{3}$ & $4,58 \pm 0,22$ & $5,59 \pm 0,29^{3}$ & $5,54 \pm 0,27^{3}$ \\
\hline Group PTX 35 & $4,77 \pm 0,18^{3}$ & $4,08 \pm 0,19^{1}$ & $5,39 \pm 0,29^{3 c}$ & $5,20 \pm 0,26^{3}$ & $4,76 \pm 0,20^{3}$ & $5,18 \pm 0,26^{3}$ & $5,21 \pm 0,23^{3}$ \\
\hline Group C & & & & & & & $4,37 \pm 0,21$ \\
\hline
\end{tabular}

Probability of values difference to Group C: $p<0,05-{ }^{1} ; \mathrm{p}<0,01-{ }^{2} ; \mathrm{p}<0,001-{ }^{3}$.

Probability of values difference between Group S and Group PTX 35 in the same time interval: $p<0,05-{ }^{a} ; \mathrm{p}<0,01$ - b; $\mathrm{p}<0,001-\mathrm{c}$.

Tab. 3: Average neutrophils number in the lungs \pm 2 x S.E.M.

\begin{tabular}{|l|c|c|c|c|c|c|c|}
\hline & 1 week & 2 weeks & 3 weeks & 4 weeks & 5 weeks & 8 weeks & 12 weeks \\
\hline Group S & $0,09 \pm 0,05$ & $0,07 \pm 0,04$ & $0,11 \pm 0,07$ & $0,07 \pm 0,07$ & $1,24 \pm 0,34^{\mathbf{1}}$ & $4,80 \pm 0,60^{\mathbf{3}}$ & $5,95 \pm 0,56^{\mathbf{3}}$ \\
\hline Group PTX 35 & $0,11 \pm 0,06$ & $0,21 \pm 0,07$ & $0,10 \pm 0,06$ & $0,08 \pm 0,04$ & $0,16 \pm 0,06^{\mathbf{a}}$ & $1,96 \pm 0,27^{\mathbf{3 b}}$ & $3,41 \pm 0,45^{\mathbf{3 c}}$ \\
\hline Group C & $0,46 \pm 0,11$ & & & & & & \\
\hline
\end{tabular}

Probability of values difference to Group C: $p<0,05-{ }^{1} ; \mathrm{p}<0,01-{ }^{2} ; \mathrm{p}<0,001-{ }^{3}$.

Probability of values difference between Group S and Group PTX 35 in the same time interval: $p<0,05-{ }^{a} ; p<0,01-{ }^{b}$; $\mathrm{p}<0,001-\mathrm{c}$.

at week 1 , and since week 3 , significantly lower values were measured 2 weeks after irradiation. The lower values in Group PTX 35 than in Group S were observed 3 weeks after irradiation.

\section{Pneumocytes type II measurement}

Significantly lower values of CK $18+$ cells on alveoli counted in Group C compared with Group S were measured 3 and 12 weeks after irradiation. In the Group PTX 35 compared with Group C and S, no significant differences were found.

Correlation between type II pneumocytes number and alveolar septal thickness values was counted with coefficient $r=-0,759$.

The number of neutrophil granulocytes

From week 5 after irradiation to the end of the experiment significantly higher number of neutrophils was found in Group S than in Group C. The extravasation of neutrophils in the lungs of PTX-treated rats was delayed, higher neutrophil number was observed since week 8 after irradiation, significantly lower than in Group S.

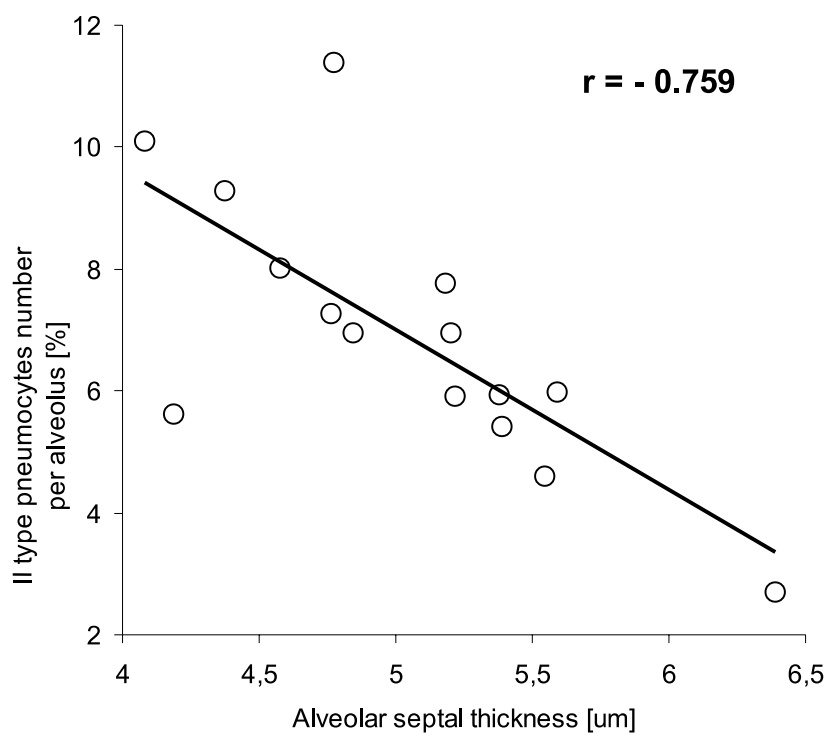

Fig. 1: Correlation between lung alveolar septal thickness and II type pneumocytes number per alveolus in percents in the lungs after a 15 Gy- $\gamma$-irradiation. 


\section{Discussion}

The type II pneumocytes are still believed to represent pneumocytar stem cells, which have ability to proliferate into a new type II pneumocytes as well as differentiate in type I pneumocytes (11). In rat lungs these cells cover approximately $3.6 \%$ while I type pneumocytes cover $96.4 \%$ of the whole alveolar surface (23). Hurley (12) suggested that type I pneumocytes check the penetration of fluid through alveolar septa rather than endothelial cells. This proves an important role of alteration of pneumocytic population kinetics in DAD by various agents including radiation. In our results we confirmed radiation-induced decrease of type II pneumocytes number 3 weeks after sublethal irradiation. It is not still clear whether increased vascular permeability may induce pneumocytes depletion or whether pneumocytes depletion may cause increased vascular permeability.

The main effect of PTX to immune reactions is to decrease the production of TNF- $\alpha(6,14)$ at the TNF- $\alpha$ protein as well as its mRNA level (2). Although Ward et al. (27) reported that PTX had only small beneficial effect on radiation-induced lung injury in rats as indicated by lung wet weight and protein content. Many other indicators of radiation-induced lung damage such as hydroxyproline content, activity of lung angiotensin converting enzyme and plasminogen activator (27), as well as the presence of interstitial edema and neutrophils (18) were positively influenced by pentoxifylline applications. The reduced alveolar septal thickness due to PTX therapy may be in relationship with TNF- $\alpha$ inhibition which can provoke pulmonary vascular endothelial injury in vitro as well as in vivo (9). PTXinduced overproduction of cAMP (2) and consequently ATP (7) may play a synergic role in potentiating surfactant secretion (8).

On the basis of our results we may hypothesize:

1. The depletion of type II pneumocytes correlates with higher alveolar septal thickness probably due to insufficient proliferation of new type II pneumocytes responsible for generation of number type I pneumocytes, which are co-responsible for the integrity of alveolar-capillary wall.

2. Pneumocytes depletion does not represent the only mechanism of higher pulmonary vascular permeability development. Other mechanisms including TNF- $\alpha$-induced damage of endothelial cells due to radiation may be involved.

3. The possible prophylactic therapy of increased pulmonary vascular permeability and subsequent interstitial lung edema could be found in the combination stimulation of type II pneumocytes proliferation with type I pneumocytes differentiation and inhibition of radiation-induced proinflammatory cytokines.

Rapid type II pneumocytes proliferation was found after the administration of keratinocyte growth factor (KGF; 24). On other hand, KGF therapy does not yield a long-term effect. Fehrenbach et al. (4) observed that new- ly generated type II pneumocytes differentiated into type I pneumocytes until day 7 after KGF intratracheal instillation application. Considering treatment of radiation-induced pulmonary vascular permeability, repeated applications would be required. Moreover, such therapy should be initiated already during the first week after irradiation to replace pool of apoptotic pneumocytes (5) by newly generated II type pneumocytes.

TNF- $\alpha$ and IL-1 (activators of proinflammatory cytokine cascade) were found in irradiated lungs $(13,20)$. Inhibitors of TNF- $\alpha$ (PTX) and of IL-1 (DXM) in combination administered, significantly ameliorate histopathological changes in rat lungs during RP manifestation (18). Moreover, their effects are only partial.

Further investigation of radiation-induced alterations of pneumocytes and possible therapy will be the matter of our future work.

\section{Conclusion}

We suggest that pneumocytes depletion is the main factor responsible for increased alveolo-capillary permeability. Non-significant effect of the pentoxifylline therapy to type II pneumocytes depletion may indicate that pneumocytes depletion is not the only mechanism increasing vascular permeability. Other mechanisms probably involve the action of proinflammatory cytokines, such as TNF- $\alpha$.

\section{Acknowledgements}

We would like to thank Mrs. Šárka Průchová, Hana Buzková, and Hana Hollerová for their skillful technical assistance.

Source: The grant No. MO 66020398127 from the Czech Republic Secretary of Defence; The grant of the Ministry of Education No. FJ MSM 111100005.

\section{References}

1. Denekamp J. Cell kinetics and radiation biology. Int J Radiat Biol 1986;49:357-

2. Doherty GM, Jensen JC, Alexander HR, Buresh CM, Norton JA. Pentoxifylline suppression of tumor necrosis factor gene transcription. Surgery 1991;110:192-8. 3. Fajardo LF. Pathology of radiation injury. $1^{\text {st }}$ ed., New York: Masson Publishing, 1982:285.

4. Fehrenbach $\mathrm{H}$, Kasper M, Tschernig T et al. Keratinocyte growth factor-induced hyperplasia of rat alveolar type II cells in vivo is resolved by differentiation into type I cells and by apoptosis. Eur Respir J 1999;14:534-44.

5. Fuks Z, Alfieri A, Haimovitz-Friedman A, Seddon A, Cordon-Cardo C. Intravenous basic fibroblast growth factor protects the lung but not mediastinal organs against radiation-induced apoptosis in vivo. Cancer J Sci Am 1995;1:62.

6. Funk JO, Ernst M, Schönharting MM, Zabel P. Pentoxifylline exerts synergistic immunomodulatory effects in combination with Dexamethasone or Cyclosporin A. Int J Immunopharmacol 1995;17:1007-16

7. Gilfillan AM, Hollingsworth M, Jones AW. The pharmacological modulation of $[3 \mathrm{H}]$ disaturated phosphatidylcholine overflow from perfused lung slices of adult rat: a new method for the study of lung surfactant secretion. Br J Pharmacol 1983;79:363-71.

8. Gobran LI, Rooney S. Adenylate cyclase-coupled ATP receptor and surfactant secretion in type II pneumocytes from newborn rats. Am J Physiol 1997;272: L187-L196.

9 Goldblum SE, Hennig B, Jay M, Yoneda K, McClain CJ. Tumor necrosis factor $\alpha$-induced pulmonary vascular endothelial injury. Infect Immun 1989;57:1218-26. 
10. Gross NJ. The pathogenesis of radiation-induced lung damage. Lung 1981;159:115-25.

11. Hasleton PS. Spencer's pathology of the lung. 5th ed., New York: McGraw-Hill, 1996:1283.

12. Hurley JV. Current views of the mechanisms of pulmonary oedema. J Pathol 1976;125:59-79.

13. Johnston CJ, Piedboeuf B, Rubin P, Williams JP, Baggs R, Finkelstein JN. Early and persistent alterations in the expression of Interleukin- $1 \alpha$, Interleukin- $1 \beta$ and Tumor necrosis factor $\alpha$ mRNA levels in fibrosis-resistant and sensitive mice af ter thoracic irradiation. Radiat Res 1996;145:762-7.

14. Mándi Y, Farkas G, Ocsovsky I, Nagy Z. Inhibition of tumor necrosis factor production and ICAM - 1 expression by pentoxifylline: beneficials effects in sepsis syndrome. Res Exp Med 1995;195:297-307.

15. Koo DJ, Yoo P, Cioffi WG, Bland KI, Chaudry IH, Wang P. Mechanism of the beneficial effects of pentoxifylline during sepsis: maintenance of adrenomedullin responsiveness and downregulation of proinflammatory cytokines. J Surg Res 2000;91:70-6.

16. Noel C, Copin MC, Hazzan M et al. Immunomodulatory effect of pentoxifylline during human allograft rejection: involvement of tumor necrosis factor-alpha and adhesion molecules. Transplantation 2000;69:1102-7.

17. Österreicher J, Vávrová J, Knížek J, Nožička J, Petýrek P. Radiation pneumonitis: The model of interstitial edema. Exp Toxicol Pathol 1999;51:491-3.

18. Österreicher J, Vávrová J, Knížek J, Nožička J, Petýrek P. Radiation Pneumonitis: The Influence of Pentoxifylline and Dexamethasone on the Alveolar Septa Thickness and the Amount of Neutrophil Granulocytes. Voj Zdrav Listy-Supplementum 1999;68:36-40.

19. Peterson TC, Neumeister M. Effect of pentoxifylline in rat and swine models of hepatic fibrosis: role of fibroproliferation in its mechanism. Immunopharmacology 1996;31:183-93.

20. Rubin P, Johnston CJ, Williams JP, McDonald S, Finkelstein JN. A perpetual cascade of cytokines postirradiation leads to pulmonary fibrosis. Int J Radiat Oncol Biol Phys 1995;33:99-109.
21. Silva JC, Rocha MF, Lima AA, Brito GA, de Menezes DB, Rao VS. Effects of pentoxifylline and nabumetone on the serum levels of IL-1beta and TNFalpha in rats with adjuvant arthritis. Inflamm Res 2000;49:14-19.

22. Travis EL. The sequence of histological changes in mouse lungs after single doses of X-rays. Int J Radiat Oncol Biol Phys 1980;16:345-7.

23. Travis EL, Tucker SL. The relationship between functional assays of radiation response in the lung and target cell depletion. Br J Cancer 1986;53:304-19.

24. Ulich TR, Yi ES, Longmuir $\mathrm{K}$ et al. Keratinocyte growth factor is a growth facto for type II pneumocytes in vivo. J Clin Invest 1994;93:1298-1306.

25. Vojtesek B, Staskova Z, Nenutil R et al. Monoclonal antibodies recognizing different epitopes of cytokeratin No.18. Folia Biol (Praha) 1989;35:373-82.

26. Ward HE, Kemsley L, Davies L, Holecek M, Berend N. The pulmonary response to sublethal thoracic irradiation in the rat. Radiat Res 1993;136:15-21.

27. Ward WF, Kim YT, Molteni A, Ts'ao C, Hinz JM. Pentoxifylline does not spare acute radiation reactions in rat lung and skin. Radiat Res 1992;129:107-11.

Submitted November 2000

Accepted January 2001.

MUDr. Jan Österreicher, Ph.D., Purkyně Military Medical Academy, Department of Radiobiology and Immunology, Třebešská 1575, 50001 Hradec Králové, Czech Republic. e-mail: osterreicher@pmfhk.cz 